\title{
Investigación sobre ciberperiodismo en España. Trayecto hacia la consolidación
}

\author{
Por Pere Masip y Josep-Lluís Micó-Sanz
}

\begin{abstract}
Resumen: Se revisa la evolución de la investigación sobre ciberperiodismo en España desde mediados de los noventa hasta la actualidad. Se traza un repaso de la investigación en la materia y se describen los principales grupos y líneas de investigación. Igualmente se detallan los altibajos sufridos por la disciplina a lo largo del período de análisis y sus consecuencias en el estado de la investigación actual y futura.

Palabras clave: Ciberperiodismo, Periodismo digital, Investigación, Grupos de investigación, España.

Title: Research on online journalism in Spain. The path towards consolidation

Abstract: We review the development of research in Online Journalism in Spain from the mid-90s to the present, presenting an overview of research carried out on this subject and describing the main research groups. The ups and downs of research and their effect on the state of current and future research are also detailed.
\end{abstract}

Keywords: Online Journalism, Research, Research groups, Spain.

Masip, Pere; Micó-Sanz, Josep-Lluís. "Investigación sobre ciberperiodismo en España. Trayecto hacia la consolidación". El profesional de la información, 2010, noviembre-diciembre, v. 19 , n. 6 , pp. $577-584$.

DOI: 10.3145/epi.2010.nov.03

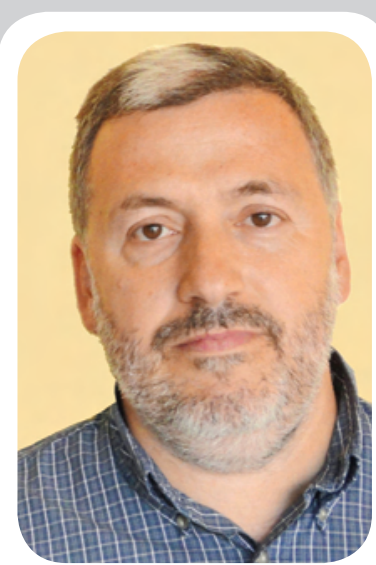

Pere Masip es doctor en periodismo por la Univ. Ramon Llull, licenciado en Geografía e Historia y diplomado en Biblioteconomía y Documentación por la UB. Es profesor en la Facultat de Comunicació Blanquerna, donde codirige el Grupo de investigación Digilab. Es autor de diversas monografías y artículos sobre periodismo digital y sobre la incidencia de las tecnologías digitales en la práctica periodística.



Josep-Lluís Micó-Sanz es periodista y profesor de la Facultat de Comunicació Blanquerna de la Universitat Ramon Llull, donde también es coordinador del Grado de periodismo y codirige el Grupo de investigación Digilab. Doctor por la Universidad Politécnica de Valencia, es autor de diversos libros y artículos sobre la digitalización de los medios y los nuevos perfiles profesionales en el sector de la información.

\section{Presentación}

El 1 de abril de 1995 el periódico Avui ponía en marcha su edición digital e inauguraba la era de los cibermedios en España. A partir de ese momento y de forma más o menos sistemática, el resto de cabeceras, desde las más modestas a los buques insignia de los grandes grupos de comunicación, empezaron su andadura por la Red. Al mismo tiempo se inauguraba una prolífica tradición de investigación en ciberperiodismo, una producción académica que Javier Díaz-Noci no duda en equiparar a la anglosajona (Díaz-Noci, 2008).

En 1996 se publicaba la que se podría considerar la primera monografía exclusivamente dedicada al periodismo en línea: El periodismo electrónico. Información y servicios multimedia en la era del ciberespacio (Armañanzas; Díaz-Noci; Meso-Ayerdi, 1996). Desde entonces, los títulos sobre el periodismo en la Red no han dejado de aparecer. Cierto es que con anterioridad a la publicación de la obra de los profesores de la Universidad del País Vasco ya se habían producido algunas aportaciones que apuntaban hacia las nuevas maneras de consumir y producir información por vía telemática. Entre ellas, conviene destacar las de Rodríguez-Delas-Heras (1991) o Pérez-Luque y Fernández-Arroyo, 1995).

"En abril de 1995 el periódico Avui ponía en marcha su edición digital e inauguraba la era de los cibermedios en España"

Rápidamente el número de investigadores sobre periodismo en internet se incrementó de forma considerable. A los ya indicados Díaz-Noci y Meso-Ayerdi 




El periodismo electrónico, de

Armañanzas, Díaz-Noci y Meso
(1997, 1999) o Pérez-Luque (1998), se les añadieron nuevos nombres como Fuentes (1997), Parra-Valcarce y Álvarez-Marcos (1997), Armentia, Elexgaray y Pérez (1999), Canga, Coca y MartínezRivera (2000), Cabrera (2000), Flores-Vivar y MiguelUrruti (2001) o Edo (2002), entre otros. Todos estos trabajos tenían un marcado carácter fundacional y básicamente eran aproximaciones descriptivas con una clara voluntad de síntesis. En ellas se trazaban las características del nuevo medio y se subrayaban las enormes posibilidades que mediante la interactividad, la hipermedialidad o el multimedia se abrían para hacer un nuevo periodismo.

Llegados a este punto resulta interesante resaltar la particular situación que vivía la investigación del periodismo digital en Catalunya. Mientras que a escala profesional la efervescencia era máxima y había un interés real por vivir y reflexionar sobre la incidencia del nuevo fenómeno en la práctica periodística, desde el punto de vista académico nos encontramos ante prácticamente un desierto. La atención por el ciberperiodismo en la universidad catalana no llegaría hasta bien entrado el nuevo milenio gracias a investigaciones individuales, resultado de tesis doctorales (Masip; Micó-Sanz, 2008).

Esta falta de investigación no significaba que no existiese en Catalunya reflexión acerca del periodismo en internet. El vacío fue rápidamente cubierto por un colectivo de profesionales en activo que vivían en primera persona el despegue de la especialidad. En el grupo había periodistas como Luis-Ángel FernándezHermana (1998), Karma Peiró, Vicent Partal (1995, 2002) o Quim Gil.

Suya fue la iniciativa de incorporar al III Congrés de periodistes catalans celebrado en 1996 un ámbito de discusión dedicado al periodismo digital, donde se gestó el Grup de Periodistes Digitals. El desinterés que mostraba la universidad por el tema quedó evidenciado por el hecho de que únicamente hubo una aportación procedente de la academia, una comunicación firmada por un emergente grupo de investigación de la Facultat de Comunicació Blanquerna de la Universitat Ramon Llull.

\section{Primera generación de investigaciones}

En opinión de Javier Díaz-Noci (2008), los autores indicados -a los que deberían añadirse otros como Ramón Salaverría, Xosé López (López; Gago; Pereira, 2000; López; Otero, 2004; López, 2006), Pilar Diezhandino o Elvira García-De-Torres- podrían considerarse miembros de una primera generación de investigadores en ciberperiodismo, que empezaron a publicar a mediados de la década de 1990.

La aparición de monografías en los primeros años de este siglo fue constante ${ }^{1}$. Aunque todavía se mantiene una amplia producción de trabajos descriptivos con vocación de manual, empiezan a publicarse los primeros que abordan el estudio de la prensa digital desde perspectivas más concretas. Se publican interesantes obras que ofrecen visiones específicas como la del diseño, la redacción o la documentación.

Armentia y sus colaboradores (2000) se ocuparon de la relación entre diseño y periodismo electrónico. En cuanto a la redacción periodística, destacan los textos de Díaz-Noci (2002) y Salaverría (2005b). Entre ambos títulos, se publica uno de los clásicos del ciberperiodismo en España: Manual de redacción ciberperiodística (Díaz-Noci; Salaverría, 2003). En esta obra los coordinadores trazan un mapa didáctico del periodismo digital con las aportaciones de casi una veintena de autores. El trabajo, además de su indudable in-

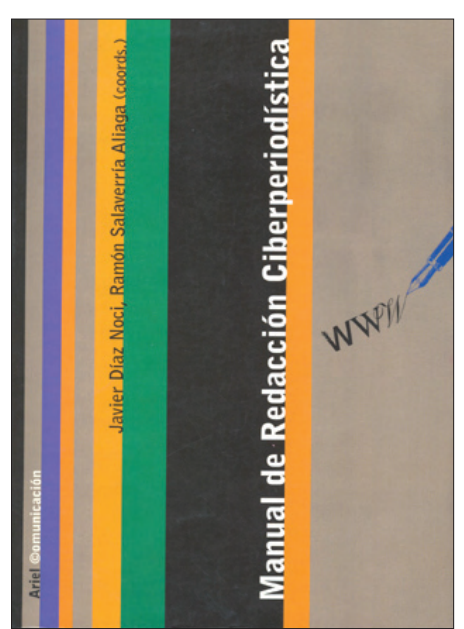

Manual de redacción ciberperiodística, de Díaz-Noci y Salaverría (coords.) terés académico, permitió la configuración de una de las redes de investigación sobre ciberperiodismo más fructíferas, constituida originalmente por profesores de las universidades de Santiago de Compostela, Navarra y el País Vasco.

\subsection{Aportaciones desde la documentación}

Mención aparte merecen los trabajos desde el campo de la documentación, que se iniciaron a finales de los años noventa y se han mantenido hasta la actualidad. M. Eulàlia Fuentes, que ya había publicado en 1997 La información en internet, se centró en el análisis de los servicios de valor añadido -entre otros, las hemerotecas digitales-, una línea que culminó con la tesis doctoral de Àngels Jiménez: Estudi de la gestió documental de la informació digital en els serveis de valor afegit als mitjans de comunica- 




Prensa digital y bibliotecas, de Abadal y Guallar ció a internet (2001). Antes, Lluís Codina había escrito $\mathrm{El} l l i$ bre digital. Una exploració sobre la informació electrónica $i$ el futur de l'edició (1996) que, aunque no es un trabajo sobre periodismo digital, aborda por primera vez en España de forma sistemática conceptos cruciales de la especialidad, como el hipertexto. La obra cuenta con una edición revisada en español publicada en 2000. También provienen de facultades de documentación las aportaciones de Juan-Carlos Marcos-Recio (1999a) y Rosana López-Carreño (2003); así como las posteriores de María Rubio-Lacoba (2007), basada en su tesis doctoral de 2004; Amparo Frías (2007) o la más reciente de Ernest Abadal y Javier Guallar (2010).

\section{Segunda generación de investigaciones}

A los autores de la primera generación pronto se les sumaría, que no sustituiría, una segunda generación de académicos más jóvenes que iniciaron su andadura investigadora unos diez años después -sobre 2005- fundamentalmente con la realización de sus tesis doctorales. Dado su origen, las contribuciones de esta segunda gene-

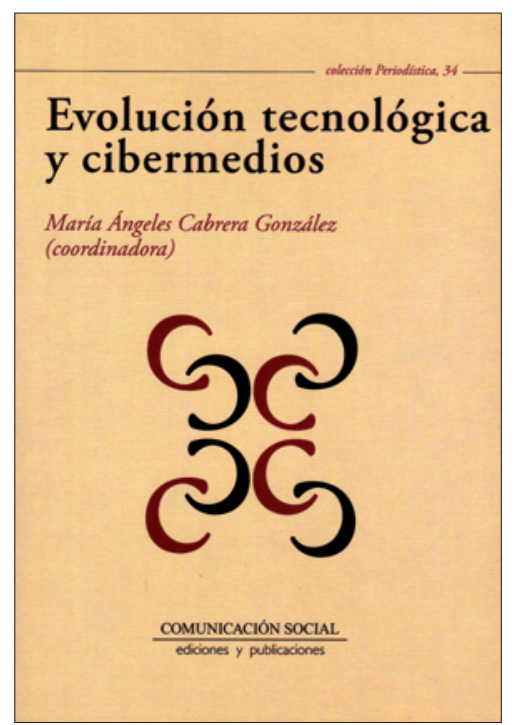

Evolución tecnológica y cibermedios, de Cabrera (coord.) ración ahondarían en aspectos todavía más concretos del periodismo digital.

La lista de esta segunda hornada de investigadores y publicaciones es amplia y, entre muchos otros, se debe incluir a Luzón (2003), Rojo (2003), Palomo (2004), Aguado (2004), Flores-Vivar y Aguado (2005), López-García (2005a，2005b，2008a，2008b), MicóSanz (2006), Domingo (2004), Almirón y Jarque
(2007), Tejedor(2007),

Scolari et al. (2007), Masip (2008), Masip y Rom (2005), Larrondo y Serrano (2007), Larrondo (2009), Masip y Micó-Sanz (2010). Paralelamente, profesores consolidados mantenían su actividad editorial: Cerezo y Zafra (2003), Edo (2003), Salaverría y Sádaba (2004), Sala$=$

\section{CONVERGENCIA DIGITAL}

Convergencia digital, de López y Pereira (coords.)

quet et al. (2006), Meso-Ayerdi (2006), Díaz-Noci et al. (2007), Salaverría y Negredo (2008), Palacios y Díaz-Noci (2009), Diezhandino (2008, 2009), Cabrera (2010), López y Pereira (2010).

Cabe agregar también la constante, aunque fluctuante, producción de los mencionados Partal o Fernández-Hermana y las de otros más recientes como Alberto Cairo (2008), con un relevante trabajo sobre la infografía, o Amaral y Gimeno (2010) sobre diseño de cibermedios. Sin embargo, los profesionales tienden a difundir sus trabajos u opiniones desde su medio natural, internet, habitualmente en weblogs: Arsenio Escolar, Juan Varela, Mario Tascón, Ismael Nafría, etc.

\section{"La presencia del ciberperiodismo en la universidad ha sido escasa y relegada a un papel secundario"}

Muchos de los autores indicados no sólo aportan nuevo conocimiento a la disciplina, sino que incorporan nuevos métodos de estudio, especialmente de carácter etnográfico. Hasta el momento, se conocía qué se hacía en la prensa digital, pero se sabía poco sobre cómo y quién lo hacía. Las tesis de Virginia Luzón (2001), Pere Masip (2006) y David Domingo (2006) y sus obras posteriores avanzaron en esa dirección. Aunque utilizando distinta metodología, habría que subrayar también algunos trabajos encaminados a conocer la situación profesional y las condiciones laborales de los periodistas digitales, entre ellos, los promovidos por el Grup de Periodistes Digitals y el Sindicat de Periodistes de Catalunya (2003). 


\section{Grupos y proyectos de investigación}

La lenta incorporación de la enseñanza del ciberperiodismo en las facultades de comunicación y la consolidación de algunos investigadores en sus respectivas universidades supuso la constitución de los primeros grupos de investigación estables. El Laboratorio de Comunicación Multimedia (MMLab), creado en 1996 y dirigido por Ramón Salaverría, fue el pionero. Le siguieron otros equipos como el grupo Novos Medios, de la Universidad de Santiago de Compostela, capitaneado por Xosé López; Infolke, de la Universidad del País Vasco, con Javier Díaz-Noci a la cabeza ${ }^{2}$ y en la actualidad con Koldo Meso-Ayerdi y José Larrañaga como investigadores principales; o el Labcom, de M. Ángeles Cabrera, en la Universidad de Málaga. La lista se podría ampliar con el Observatorio de Investigación en Medios Digitales (Oimed) dirigido por Elvira García-De-Torres; el Observatorio de Periodismo en Internet (OPEI) con Jesús Flores-Vivar como coordinador; el Grup de Recerca d'Interaccions Digitals (GRID), vinculado a la Universitat de Vic; el Grupo de Investigación de los Medios Digitales Valencianos, de Guillermo López; o el Digilab de la Universidad Ramon Llull codirigido por Josep-Lluís Micó-Sanz y Pere Masip.

La proliferación de investigadores y grupos, además de un evidente aumento de la producción, tiene otras consecuencias relevantes que ponen de manifiesto que se trata de una disciplina emergente. Así, proyectos centrados en el ciberperiodismo reciben el apoyo financiero del Ministerio de Educación y Ciencia (en cualquiera de sus denominaciones) mediante los planes nacionales de $\mathrm{I}+\mathrm{D}+\mathrm{i}$, se defienden tesis doctorales sobre el asunto y surgen los primeros encuentros académicos.

El desarrollo del ciberperiodismo como objeto de investigación se tradujo en la concesión de financiación para proyectos sobre este tema en convocatorias públicas competitivas. Entre ellos destaca el proyecto El impacto de internet en los medios de comunicación en España (BSO2002-04206-01,-02,-03,-04), con financiación del Ministerio de Ciencia y Tecnología, que contó con la participación de investigadores de las universidades de Navarra, País Vasco, Santiago y Málaga. El proyecto dio como resultado, entre otros, la monografía Cibermedios (Salaverría, 2005a), en la que se arroja luz sobre el proceso de consolidación de los diarios digitales en España. Algunos de los investigadores del proyecto simultáneamente formaron parte de la acción europea COST A20 The impact of the internet on the mass media in Europe, en cuyo marco se organizó en Pamplona el congreso Towards new media paradigms (Salaverría; Sádaba, 2004). En 2003, recibió igualmente apoyo económico el proyecto impulsado por Elvira García-De-
Torres, del CEU-San Pablo, que tenía como objetivo detectar tendencias en el escenario de los nuevos medios en España y Latinoamérica ${ }^{3}$.

El interés por el ciberperiodismo también ha estado presente en las convocatorias de los dos últimos planes nacionales: $2004-2007$ y 2008-2011 ${ }^{4}$. En concreto, entre 2004 y 2009 han sido concedidos, al menos, 12 proyectos ${ }^{5}$ sobre medios digitales. Ocho de ellos son coordinados y han sido liderados por los mismos investigadores que llevaron a cabo El impacto de internet en los medios... referido anteriormente ${ }^{6}$. Junto a ellos, en 2006 obtuvieron financiación sendos proyectos dirigidos por Elvira García-De-Torres y Rosa Franquet; y en 2007, Mariano Cebrián-Herreros ${ }^{7}$. El primero de ellos se puede considerar una continuación de otro señalado anteriormente, mientras que el segundo centra su atención en la televisión, si bien ofrece un punto de conexión con el ciberperiodismo ${ }^{8}$. En la convocatoria de 2009, la línea de investigación del equipo de Franquet recibió de nuevo financiación pública?

\section{"La investigación sobre ciberperiodismo ha adquirido unos niveles de producción y calidad notables"}

\section{Encuentros académicos}

En cuanto a los encuentros académicos, ya nos hemos referido al III Congrés de periodistes catalans, que en noviembre de 1996 dedicaba diversas sesiones a debatir cómo serían las redacciones digitales, las nuevas fuentes informativas, las necesidades formativas y de reciclaje de los periodistas ante la revolución digital... A partir de ese momento los congresos sobre el tema se sucedieron, aunque pocos consiguieron consolidarse en el tiempo. Ese mismo año la Universidad de Navarra organizaba el I Congreso internacional de los medios de comunicación en internet, con el objetivo de analizar cómo elaborar la información para la Red y el nuevo perfil de los periodistas. El congreso tuvo dos ediciones más.

El Grup de Periodistes Digitals organizó en 1998 en Barcelona el I Congreso internacional de publicación electrónica, y al año siguiente se celebraba el I Congrés internacional comunicar a l'era digital (Franquet; Larrégola, 1999). Ninguno de ellos gozó de nuevas ediciones. Todo lo contrario sucedió con el Congreso nacional de periodismo digital que se abría en 2000 y que se ha mantenido fiel a su cita anual en Huesca hasta la actualidad. Su éxito hay que buscarlo en la mesurada 


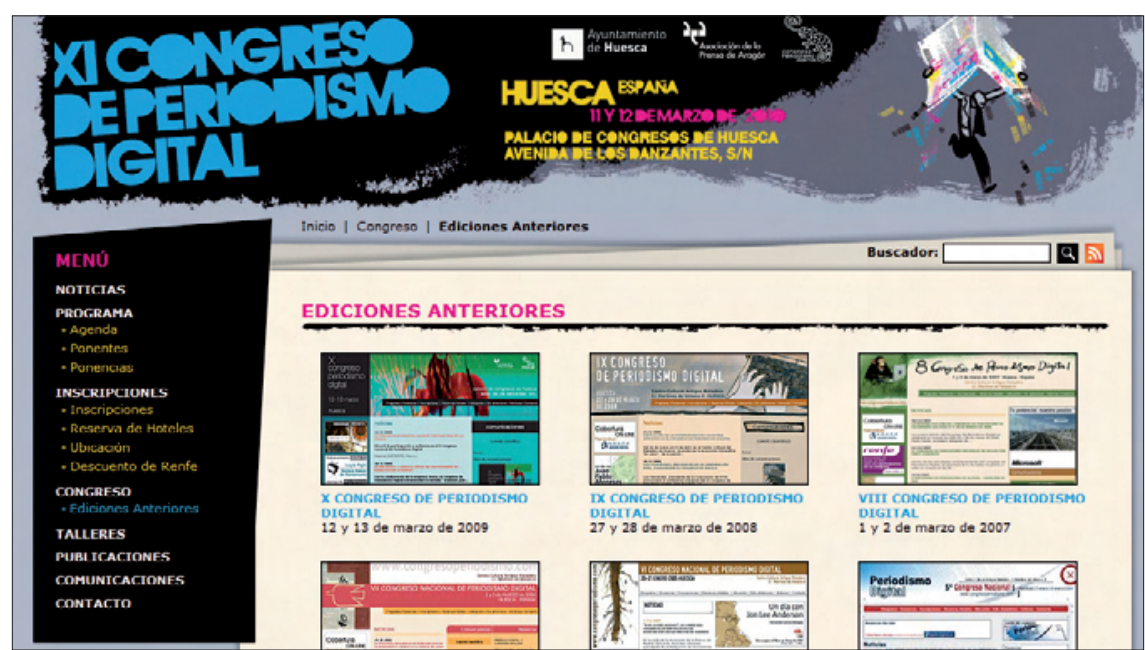

Web del XI Congreso de periodismo digital

combinación de aportaciones profesionales con otras académicas, ofreciendo un foro de debate común para que unos y otros intercambien visiones del periodismo digital. Sin lugar a dudas, ha sido durante años un referente en España.

El número de citas relacionadas con el ciberperiodismo se multiplicó en los primeros años de la década de 2000, aunque pocas han conseguido consolidarse. Sin carácter exhaustivo, cabe añadir el Congreso internacional de ciberperiodismo que la Universidad Antonio de Nebrija acogió entre 2003 y 2006, el Congreso internacional de blogs y periodismo en la Red de la Universidad Complutense de Madrid (2006-) o, el más reciente Congreso internacional de ciberperiodismo y web 2.0 (2009-), organizado por Koldo Meso-Ayerdi en la Universidad del País Vasco.

Junto a los congresos indicados, de corte fundamentalmente académico, también han proliferado, al estilo del congreso de Huesca, otros foros de debate que aúnan la perspectiva profesional y la académica. Entre ellos destacan la E-week: el Seminari internacional de periodisme digital (Vilanova i la Geltrú, Barcelona, 2003-2007), Setmana digital de Vic (Barcelona, 2004-) o el itinerante Congreso internacional de nuevo periodismo, impulsado por el periodista Fernando Jáuregui.

\section{Tesis doctorales}

Desde 1999, momento en que M. Ángeles Cabrera defiende la primera tesis doctoral específicamente dedicada al periodismo digital, hasta la actualidad (junio 2010), se han realizado 52 trabajos $^{10}$. La media anual es de poco más de 4,5 tesis sobre ciberperiodismo y alcanza su máximo en 2006, con 8, seguido de 2005 con 7, lo que supone un $30 \%$ de los trabajos presentados.

Aunque no han faltado tesis de tipo descriptivo generalista, la tendencia ha sido, como no puede ser de otro modo, a la especialización. Desde los inicios ha habido trabajos que abordan aspectos concretos del ciberperiodismo, del concepto de noticia (Machado, 2000; Batista, 2001) a la interactividad (Cebrián, 2005; Rost, 2006; Murelaga, 2007; Meseguer, 2008), pasando por la hipertextualidad/ hipermedialidad (Pérez-Marco, 2003; Larrondo, 2008) o la usabilidad (Cobo, 2005; Lerma-Noriega, 2005), la documentación informativa (Marcos-Recio, 1999b; Jiménez, 2001; Rubio-Lacoba, 2004), las rutinas productivas (Luzón, 2001; Palomo, 2002; Domingo, 2006; Masip, 2006) o las nuevas formas de comunicación (López-Carreño, 2003; Alonso, 2004; Sánchez-González, 2007), por poner sólo algunos ejemplos.

Prácticamente la mitad, 23 de las 52 tesis, han sido defendidas en la Universidad Complutense de Madrid (12) y en la Autónoma de Barcelona (11). El resto se lo reparten otras 15 universidades: Málaga, La Laguna y Navarra, 4 tesis; 3 en la del País Vasco; 2 en las de Murcia, Pontificia de Salamanca y CEU-San Pablo; y 1 en las de Barcelona, Católica San Antonio, Granada, Internacional de Catalunya, Pompeu Fabra, Ramon Llull, Sevilla y Zaragoza.

En cuanto a los directores se observa una gran dispersión. Únicamente Bernardo Díaz-Nosty ha dirigido 4 tesis; De-Pablos, Franquet, González-Antón, Lallana, Rodrigo-Alsina y Martínez-Vallvey han dirigido 2; y otros 39 se reparten el resto ${ }^{11}$. Las cifras ponen de manifiesto la falta de referentes claros en la universidad española. De hecho, ninguno de los directores que han asumido dos o más trabajos han convertido el ciberperiodismo en su ámbito de especialización.

\section{"El número de grupos e investigadores se ha multiplicado, y las tesis se han disparado"}

\section{Conclusiones}

A pesar de la relativa juventud de la disciplina, la investigación sobre ciberperiodismo ha adquirido unos niveles de producción notables y una elevada calidad. En poco tiempo, el número de grupos e investigadores se ha multiplicado, el número de tesis se ha disparado y los congresos sobre la materia han proliferado -algunos están plenamente consolidados-. Con todo, ciertos 
indicadores permiten entrever que la investigación en ciberperiodismo está todavía en fase de consolidación, con unas bases menos sólidas de lo que las cifras y ejemplos ofrecidos en este artículo permitirían deducir. Resulta revelador que los profesores que han dirigido más tesis no sean especialistas en la materia, mientras que los principales investigadores prácticamente no aparecen en las listas de directores.

Los motivos que explican esta situación hay que buscarlos en el encaje de la disciplina en la universidad. Como puso de manifiesto Santiago Tejedor en su tesis doctoral (2006), la presencia del ciberperiodismo en la universidad ha sido escasa y relegada a un papel secundario. Sólo un 7\% de las materias de los programas de las licenciaturas en periodismo están vinculadas directa o indirectamente al ciberperiodismo. A ello, habría que sumar la actitud adoptada por parte del cuerpo docente de los centros, que en su día no mostró especial interés por el fenómeno. Los profesores en una situación académica consolidada deberían haber potenciado la incorporación de la materia en los planes de estudio y liderado la investigación. Desde su situación privilegiada estaban en condiciones de intentarlo, pero, con algunas excepciones, no lo hicieron. José-Manuel Pérez-Tornero lo atribuye a la actitud acomodaticia de ciertos sectores de profesores, que reniegan de un fenómeno que consideran pasajero y que puede llegar a poner en cuestión su manera de ejercer la docencia (2007). Las exigencias de la disciplina, la falta de reconocimiento o la necesidad de reciclaje permanente parecen argumentos antagónicos y poco compatibles con la comodidad de la que disfrutaban algunos. La consecuencia fue la previsible: las asignaturas sobre ciberperiodismo eran escasas y su docencia recayó en jóvenes profesores, cargados de ilusiones, pero con poca experiencia, una situación laboral inestable y una carrera docente por labrar. Ha sido particularmente esta primera generación, a la cual se han añadido con posterioridad nombres de la segunda generación que identifica Díaz-Noci, la que ha impulsado la investigación en ciberperiodismo y la que la ha encumbrado a niveles relevantes.

\section{Notas}

1. Debido al ingente volumen de producción sobre ciberperiodismo, para este trabajo nos referiremos sólo a monografías y no haremos mención a contribuciones en forma de artículos o comunicaciones y ponencias a congresos.

2. Desde 2009 Javier Díaz-Noci está vinculado a la Universitat Pompeu Fabra, en Barcelona.

3. Proyecto Medios de comunicación en el ciberespacio. El impacto de internet en las estructuras comunicativas tradicionales. El nuevo entorno digital español. Tendencias (BSO2003-08535).

4. Los resultados de las convocatorias anteriores no están disponibles en la web del Ministerio de Educación y Ciencia.

5. Datos obtenidos del Ministerio de Ciencia e Innovación (Micinn) para las convocatorias de 2004 a 2008. La relación de los proyectos aprobados en la convocatoria 2009 no estaba disponible en la web en el momento de escribir este artículo (junio 2010), por lo que la información puede ser parcial. http://ciencia.micinn.fecyt.es

6. Se trata de los proyectos coordinados: Convergencia digital en los medios de comunicación, financiado por el Ministerio de Ciencia y Tecnología (2006-2008) (SEJ2006-14828-C06-08), y Evolución de los cibermedios españoles en el marco de la convergencia (2009-10) (CSO2009-13713-C0501,-02,-03,-04), con el apoyo del Ministerio de Ciencia e Innovación.

7. Cybermedia: innovaciones, procesos y nuevos desarrollos del periodismo en internet, telefonía móvil y otras tecnologías del conocimiento (SEJ200767138).

8. Proyectos: Medios de comunicación en el ciberespacio. El impacto de internet en los medios de comunicación tradicionales. El nuevo entorno digital español y latinoamericano. Tendencias (2007-2010) (SEJ2006-15495) y Televisión interactiva en el entorno cross media: tipología de la oferta, los contenidos, los formatos y los servicios emergentes (SEJ2006-11245).

9. Entorno cross media: transformaciones organizativas y productivas en los grupos radiotelevisivos (CSO2009-09367)

10. Para determinar la cifra de tesis doctorales sobre ciberperiodismo se ha consultado la base de datos Teseo, realizando búsquedas combinadas en los campos título y resumen a partir de los términos siguientes: ciberperiodismo, periodismo, periódico, periodística, prensa, web, portal, noticia, géneros, información, internet, online, digital, electrónico. La lista obtenida se ha completado con la consulta complementaria de repositorios de tesis doctorales (Biblioteca Virtual Miguel de Cervantes y TDX) y con el conocimiento de la especialidad de los propios autores. A pesar de la teórica exhaustividad de Teseo, se da la circunstancia de que algunas de las tesis doctorales sobre ciberperiodismo localizadas por los autores no aparecen referenciadas en dicha base de datos. La última consulta para la elaboración de este artículo se realizó en junio de 2010, por lo que no se incluyen otras tesis leídas con posterioridad, como la defendida por Ana Serrano en la Universidad del País Vasco sobre el diseño de nodos iniciales en los cibermedios.

11. La suma total de doctores es de 55 debido a que en diversos casos hay dos directores.

\section{Bibliografía}

Abadal, Ernest; Guallar, Javier. Prensa digital y bibliotecas. Gijón: Trea, 2010 .

Aguado, Juan-Miguel. E-Comunicación. Dimensiones sociales y profesionales de la comunicación en los nuevos entornos tecnológicos. Sevilla: Comunicación Social, 2004.

Almirón, Núria; Jarque, Josep-Manuel. El mito digital. Discursos hegemónicos sobre internet y periodismo. Barcelona: Anthropos, 2007.

Alonso, Jaime. Análisis descriptivo de nuevos medios en internet: diarios digitales, portales, buscadores y directorios, bitácoras o weblogs, redes ciudadanas y comunidades virtuales. Tesis doctoral. Universidad Pontificia de Salamanca, 2004.

Amaral, Franciso; Gimeno, Daniel. Evolución, tendencias y modelos en el diseño de webs de noticias. Barcelona: Sol90media, 2010.

Armañanzas, Emy; Díaz-Noci, Javier; Meso-Ayerdi, Koldo. El periodismo electrónico. Información y servicios multimedia en la era del ciberespacio. Barcelona: Ariel, 1996.

Armentia, José-Ignacio; Caminos, José-María; Elexgaray, Jon; Martín, Flora; Merchán, Iker. El diario digital. Análisis de los contenidos textuales, aspectos formales y publicitarios. Barcelona: Bosch, 2000.

Armentia, José-Ignacio; Elexgaray, Jon; Pérez, Juan-Carlos. Diseño y periodismo electrónico. Bilbao: Servicio Editorial de la Universidad del País Vasco, 1999.

Batista, Noemí. Características de la noticia en internet y análisis de su situación actual. Los casos de EE.UU. y España. Tesis doctoral. Universidad de Navarra, 2001.

Cabrera, M. Ángeles (coord.). Evolución tecnológica y cibermedios. Sevi1la: Comunicación Social, 2010.

Cabrera, M. Ángeles. Evolución y tendencias en la configuración gráfica de la prensa "online". Tesis doctoral. Universidad de Málaga, 1999. 
Cabrera, M. Ángeles. La prensa online. Los periódicos en la www. Barcelona: Cims, 2000.

Cairo, Alberto. Infografía 2.0. Visualización interactiva de información en prensa. Madrid: Alamut, 2008.

Canga, Jesús; Coca, César; Martínez-Rivera, Eloy. Diarios digitales. Apuntes sobre un nuevo medio. Bilbao: Servicio Editorial de la Universidad del País Vasco, 2000.

Cebrián, Marta. Información interactiva sobre radio y televisión en internet. Tesis doctoral. Universidad Complutense de Madrid, 2005.

Cerezo, José-Manuel; Zafra, Juan-Manuel. El impacto de internet en la prensa. Madrid: Fundación Auna, 2003.

Cobo, Juan-Cristóbal. Organización de la información y su impacto en la usabilidad de las tecnologías interactivas. Tesis doctoral. Universitat Autònoma de Barcelona, 2005.

Codina, Lluís. El libro digital y la www. Barcelona: Tauro, 2000.

Codina, Lluís. El llibre digital. Una exploració sobre la informació electrònica i el futur de l'edició. Barcelona: Centre d'Investigació de la Comunicació, 1996

Díaz-Noci, Javier. "Ciberperiodismo, investigación y docencia: una oportunidad única”. Anàlisi, 2008, n. 36, pp. 41-51.

http://www.raco.cat/index.php/Analisi/article/view/94509/119724

Díaz-Noci, Javier. La escritura digital. Hipertexto y construcción del discurso informativo en el periodismo electrónico. Bilbao: Servicio Editoria de la Universidad del País Vasco, 2002.

Díaz-Noci, Javier; Larrañaga-Zubizarreta, José; Larrondo, Ainara Meso-Ayerdi, Koldo. El impacto de internet en los medios de comunicación vascos. Bilbao: Servicio Editorial de la Universidad del País Vasco, 2007.

Díaz-Noci, Javier; Meso-Ayerdi, Koldo. Medios de comunicación en internet. Madrid: Anaya Multimedia, 1997.

Díaz-Noci, Javier; Meso-Ayerdi, Koldo. Periodismo en internet. Modelos de la prensa digital. Bilbao: Servicio Editorial de la Universidad del País Vasco, 1999.

Díaz-Noci, Javier; Salaverría, Ramón (coords.). Manual de redacción ciberperiodística. Barcelona: Ariel, 2003.

Diezhandino, M. Pilar. Criterio noticioso. Madrid: Prentice Hall, 2009.

Diezhandino, M. Pilar (dir.). Periodismo en la era de internet. Claves para entender la situación actual de la información periodística en España. Barcelona: Ariel; Madrid: Fundación Telefónica, 2008.

Domingo, David. Inventing online journalism. Development of the internet as a news medium in four Catalan online newsrooms. Tesis doctoral. Universitat Autònoma de Barcelona, 2006

Domingo, David. Periodisme digital a Catalunya. Radiografia de 445 webs informatius. Barcelona: Col-legi de Periodistes de Catalunya, 2004.

Edo, Concha. Del papel a la pantalla. La prensa en internet. Sevilla: Comunicación Social, 2002.

Edo, Concha. Periodismo informativo e interpretativo. El impacto de internet en la noticia, las fuentes y los géneros. Sevilla: Comunicación Social, 2003.

Fernández-Hermana, Luís-Ángel. En.red.ando. Barcelona, Ediciones B, 1998.

Flores-Vivar, Jesús; Aguado, Guadalupe. Modelos de negocio en el ciberperiodismo: estrategias de los medios para el desarrollo de negocios en la red. Madrid: Fragua, 2005

Flores-Vivar, Jesús; Miguel-Urruti, Alberto. Ciberperiodismo. Nuevos enfoques, conceptos y profesiones emergentes en el mundo infodigital. Madrid: Ediciones 2010 SL, 2001.

Franquet, Rosa; Larrégola, Gemma (eds.). Comunicar a l'era digital. Barcelona: Societat Catalana de Comunicació, 1999.

Franquet, Rosa; Soto, Maite; Ribes, Xavier; Fernández-Quijada, David Assalt a la xarxa: la batalla decisiva dels mitjans de comunicació on-line en català. Barcelona: Col.legi de Periodistes de Catalunya, 2006.

Frías, Amparo. Els estudis d'usuaris en els serveis personalitzats als mi- tjans de comunicació a internet. Tesis doctoral. Universitat de Barcelona, 2007

Fuentes, M. Eulàlia. La información en internet. Barcelona: Cims, 1997.

Grup de Periodistes Digitals, Sindicat de Periodistes de Catalunya. Informe sobre la situació laboral i professional dels periodistes digitals a Catalunya, 2003.

Jiménez, Àngels. Estudi de la gestió documental de la informació en els serveis de valor afegit dels mitjans de comunicació a internet: el cas de la premsa diària a l'estat espanyol. Tesis doctoral. Universitat Autònoma de Barcelona, 2001.

Larrondo, Ainara. El reportaje hipermedia. Análisis del género en los especiales de Elmundo.es, Elpais.com y Lavanguardia.es. Tesis doctoral. Universidad del País Vasco, 2008.

Larrondo, Ainara. Los géneros en la redacción ciberperiodística. Contexto, teoría y práctica actual. Bilbao: Universidad del País Vasco, 2009.

Larrondo, Ainara; Serrano, Ana (eds.). Diseño periodístico en internet. Bilbao: Servicio Editorial de la Universidad del País Vasco, 2007.

Lerma-Noriega, Claudia. La usabilidad de la prensa digital de habla hispana. Análisis de los sitios web de ABC, El mundo, El universal y La jornada. Tesis doctoral. Universidad Complutense de Madrid, 2005.

López, Xosé (coord.). Sistemas digitales de información. Madrid: Pearson Educación, 2006.

López, Xosé; Gago, Manuel; Pereira, Xosé. O novo xornalismo electrónico. Santiago de Compostela: Edicións Lea, 2000.

López, Xosé; Otero, Marita. Las herramientas tecnológicas de la nueva información periodística. A Coruña: Netbiblio, 2004.

López, Xosé; Pereira, Xosé (coords.). Convergencia digital. Reconfigura ción de los medios de comunicación en España. Santiago de Compostela: Servicio Editorial de la Universidad de Santiago de Compostela, 2010.

López-Carreño, Rosana. Análisis de los portales periodísticos españoles. Taxonomía de sus elementos componentes. Tesis doctoral. Universidad de Murcia, 2003

López-García, Guillermo (ed.). Comunicación local y nuevos formatos periodísticos en internet: cibermedios, confidenciales y weblogs. València: Servei de Publicacions de la Universitat de València, 2008a. http://www.cibermediosvalencianos.es/ComunicacionLocal.pdf

López-García, Guillermo (ed.). El ecosistema digital: modelos de comuni cación, nuevos medios y público en internet. València: Servei de Publicacions de la Universitat de València, 2005a.

López-García, Guillermo. Los cibermedios valencianos: cartografía, características y contenidos. València: Servei de Publicacions de la Universitat de València, 2008b.

http://www.cibermediosvalencianos.es/cibermedios.pdf

López-García, Guillermo. Modelos de comunicación en internet. València: Tirant Lo Blanch, 2005b.

Luzón, Virginia. Internet, l'eina inevitable. Periodistes, mitjans i missatges en la Xarxa. Barcelona: Col-legi de Periodistes de Catalunya, 2003.

Luzón, Virginia. La irrupción de internet en las rutinas productivas de los informativos diarios televisivos. El caso de TV3, Televisió de Catalunya. Tesis doctoral. Universitat Autònoma de Barcelona, 2001.

Machado, Elías. La estructura de la noticia en las redes digitales. Un estudio de las consecuencias de las metamorfosis tecnológicas en el periodismo. Tesis doctoral. Universitat Autònoma de Barcelona, 2000.

Marcos-Recio, Juan-Carlos. La documentación electrónica en los medios de comunicación. Madrid: Fragua, 1999a.

Marcos-Recio, Juan-Carlos. Un nuevo concepto de información y documentación en los periódicos electrónicos. Tesis doctoral. Universidad Complutense de Madrid, 1999b.

Masip, Pere. Internet a les redaccions. Informació diària i rutines periodístiques. Barcelona: Trípodos, 2008.

Masip, Pere. Presència i ús d'internet a les redaccions: periodistes, rutines professionals i tecnologia: el cas de La vanguardia, Catalunya ràdio i Televisió de Catalunya. Tesis doctoral. Universitat Ramon Llull, 2006. 
Masip, Pere; Micó-Sanz, Josep-Lluís (coords.). La convergència comunicativa a la premsa local i comarcal. Barcelona: Generalitat de Catalunya, 2010.

Masip, Pere; Micó-Sanz, Josep-Lluís. "Una aproximación a la investigación sobre ciberperiodismo en Catalunya: una asignatura pendiente". En: Congreso Fundacional de la AE-IC. Santiago de Compostela, 2008.

Masip, Pere; Rom, Josep (eds.). La utopia digital en els mitjans de comunicació. Barcelona: Trípodos, 2005.

Meseguer, Ángel. El concepto de interactividad en la radio comercial española en internet. Repercusiones en el ámbito regional murciano. Tesis doctoral. Universidad de Murcia, 2008.

Meso-Ayerdi, Koldo. Introducción al ciberperiodismo: breve acercamiento al estudio del periodismo en internet. Bilbao: Servicio Editorial de la Universidad del País Vasco, 2006.

Micó-Sanz, Josep-Lluís. Periodisme a la xarxa. Llenguatge nou d'un ofici vell. Vic: Eumo, 2006.

Murelaga, Jon. La interactividad de las radios musicales españolas en internet (2005). Tesis doctoral. Universidad del País Vasco, 2007.

Palacios, Marcos; Díaz-Noci, Javier (eds.). Online journalism: research methods. A multidisciplinary approach in comparative perspective. Bilbao: Servicio Editorial de la Universidad del País Vasco, 2009.

http://www.argitalpenak.ehu.es/p291-content/es/contenidos/libro/se_indice_ciencinfoles_ciencinf/adjuntos/journalism.pdf

Palomo, M. Bella. El periodista on-line: de la revolución a la evolución. Sevilla: Comunicación Social, 2004

Palomo, M. Bella. El uso redaccional de internet en la prensa diaria española. Tesis doctoral. Universidad de Málaga, 2002.

Partal, Vicent. Periodistes i documentalistes: on és la frontera? Barcelona: Universitat Oberta de Catalunya, 2002.

http://www.uoc.edu/web/cat/art/uoc/partal0402/partal0402_imp.html

Partal, Vicent. "The role of journalists in the new media". En: 17th Conference of the Society of Newspapers Design. Barcelona, 1995.

Parra-Valcarce, David; Álvarez-Marcos, José. Ciberperiodismo. Madrid: Editorial Síntesis, 1997.

Pérez-Luque, M. José. El periodismo y las nuevas tecnologías. Pamplona: Newbook, 1998.

Pérez-Luque, M. José; Fernández-Arroyo, Noelia. "La integración de la tecnología multimedia y de las redes interactivas: revolución de los sistemas informativos". Informe de situación. La industria de la comunicación. Bilbao: BBV, 1995, pp. 105-145.

Pérez-Marco, Sonia. El concepto de hipertexto en el periodismo digital. Análisis de la aplicación del hipertexto en la estructuración de las noticias de las ediciones digitales de tres periódicos españoles (www.elpais.es, www.elmundo.es, www.abc.es). Tesis doctoral. Universidad Complutense de Madrid, 2003.

Pérez-Tornero, José-Manuel. "Periodismo del presente-futuro". En: Tejedor-Calvo, Santiago. La enseñanza del ciberperiodismo. De la alfabetización digital a la alfabetización ciberperiodística. Sevilla: Comunicación Social, 2007, pp. 8-18.
Rodríguez-De-las-Heras, Antonio. Navegar por la información. Madrid: Fundesco, 1991.

Rojo, Pedro-Antonio. Producción periodística y nuevas tecnologías. Sevilla: Comunicación Social, 2003.

Rost, Alejandro. La interactividad en el periódico digital. Tesis doctoral. Universitat Autònoma de Barcelona, 2006.

Rubio-Lacoba, María. Documentación informativa en el periodismo digital. Madrid: Síntesis, 2007.

Rubio-Lacoba, María. La documentación informativa en el periodismo digital. Análisis del servicio documental, del documentalista y del mensaje documental en el nuevo medio digital. Tesis doctoral. Universidad Pontificia de Salamanca, 2004.

Salaverría, Ramón (coord.). Cibermedios. El impacto de internet en los medios de comunicación en España. Sevilla: Comunicación Social, 2005a.

Salaverría, Ramón. "La investigación sobre ciberperiodismo en España: tendencias, resultados y perspectivas”. En: López-García, Guillermo (ed.). Comunicación local y nuevos formatos periodísticos en internet: cibermedios, confidenciales y weblogs. València: Servei de Publicacions de la Universitat de València, 2008, pp. 15-34.

http://www.cibermediosvalencianos.es/comloc/Salaverria.pdf

Salaverría, Ramón. Redacción periodística en internet. Pamplona: Eunsa, 2005 b.

Salaverría, Ramón; Negredo, Samuel. Periodismo integrado. Convergencia de medios y reorganización de redacciones. Barcelona: Sol90media, 2008.

Salaverría, Ramón; Sádaba, Charo (eds.). Towards new media paradigms: content, producers, organisations and audiences. II International conference of COST A20. Pamplona: Eunate, 2004.

Sánchez-González, María. Cibermedios de acceso abierto con información confidencial en España. Tesis doctoral. Universidad de Málaga, 2007.

Scolari, Carlos; Jarque, José-Manuel; Perales, Cristina; Navarro, Héctor; Coll, Ignasi. El canvi digital als mitjans de proximitat: les transformacions generades per la introducció de les tecnologies digitals en les redaccions de la premsa comarcal i les ràdios i televisions locals. Barcelona: Generalitat de Catalunya, 2007.

Tejedor, Santiago. La enseñanza del ciberperiodismo. De la alfabetización digital a la alfabetización ciberperiodística. Sevilla: Comunicación Social, 2007.

Tejedor, Santiago. La enseñanza del ciberperiodismo en las licenciaturas de periodismo de España. Tesis doctoral. Universitat Autònoma de Barcelona, 2006.

Pere Masip, Josep-Lluís Micó-Sanz. Facultat de Comunicació Blanquerna, Universitat Ramon Llull. peremm@blanquerna.url.edu joseplluisms@blanquerna.url.edu

\section{Suscripción EPI sólo online}

Pensando sobre todo en los posibles suscriptores latinoamericanos, ya no es obligatorio pagar la suscripción impresa de EPI para acceder a la online.

EPI se ofrece a instituciones en suscripción "sólo online" a un precio considerablemente más reducido (90 euros/año), puesto que en esta modalidad no hay que cubrir los gastos de imprenta ni de correo postal. 